\title{
Special issue on the Security Track at the ACM Symposium on Applied Computing 2013
}

\author{
Giampaolo Bella $^{1}$ - Helge Janicke ${ }^{2}$
}

Published online: 2 April 2015

(C) Springer-Verlag Berlin Heidelberg 2015

Mobile systems are becoming more and more ubiquitous. With the number of people using mobile devices that are continuously connected to the Internet or exchange information via peering and other near-field transmission technologies, these devices are now an essential part of our social interactions in a globalised world. The use of mobile devices has increased dramatically over recent years, and in many countries the lack of traditional network infrastructures means that they are often the only way to connect to the Internet. Whilst most people still associate mobile devices with smart phones or mobile computers, there is in fact an increasing trend to other mobile computing devices such as tablets, Wi-fi-enabled cameras and a variety of smart hardware, which are also used to form ad hoc networks in our homes and offices. This proliferation, associated with our pervasive use of mobile devices, leads to new cyber security threats to these devices and hence to the data they hold and process.

Threats to mobile systems are manifold and whilst many of the security principles that traditionally have been applied to IT infrastructures still apply, they are in urgent need of review and adaption. Given the proliferation of mobile devices and the extent that these are becoming pervasive in our societies, we clearly need to revisit the threats that are created by our new inventions and lifestyle. This is the direction in which the following six contributions shed some bright light. They are significantly extended versions of the best papers presented at the Security Track of the ACM Symposium on Applied Com-

Giampaolo Bella

giamp@dmi.unict.it

1 Dipartimento di Matematica e Informatica, Università di Catania, Catania, Italy

2 Faculty of Technology, De Montfort University, Leicester, UK puting in 2013. Each of them received two ample reviews to question their contribution once more and, at the same time, to evaluate the significance of the extension to the conference version. Throughout this process, two papers were rejected. A short summary of the six accepted manuscripts follow.

Martina et al. address explicitly some of the threats mentioned above in their article "Adaptive Threat Model for Security Ceremonies". Security ceremonies extend protocol design and analysis to include human peers, and thus their analysis bears the potential to detect security flaws that technology-focused approaches could be missing. The article demonstrates the formal analysis of protocols against a dynamic threat model that can be adjusted to specified ceremonies, yielding an adaptable model for the analysis of security ceremonies for realistic scenarios.

Additional concerns may derive from how humans use their devices. A recent one for many organisations is the wide use of personal devices at the place of work. Whilst in many instances this is driven by the convenience of users, some organisations see this as a way of reducing equipment and maintenance costs. However, the use of devices that are not owned by the organisation, over which control from both a physical and administrative perspective is limited, creates additional risks. Many organisations address this risk by enforcing policies on the use of Bring Your Own Device (BYOD). In their contribution entitled "Formal Modeling and Automatic Enforcement of Bring Your Own Device Policies", Armado et al. propose a security framework for the verification and enforcement of BYOD security policies for Android devices. It requires only minor modifications at the application level and enables organisations to control the risk of allowing their workforce to use their personal devices for professional work.

Whilst BYOD is creating concerns for enterprise security, the security of the devices themselves is becoming more 
and more important to address from the viewpoint of mobile operating systems. The increase in malware that is targeting mobile operating systems, such as Android, is threatening a large user base and puts their right to privacy at risk. In "Mobile Sandbox Combining Static and Dynamic Analysis with Machine Learning Techniques", Spreitzenbarth et al. address the static and dynamic analysis of Android malware using a sandboxing approach. This automatable analysis of Android applications combined with machine learning techniques allows for more effective detections of malicious apps.

Smaller mobile devices are deployed as part of wireless sensor networks in an industrial setting and for safety critical applications. Even more than traditional mobile devices, these networks are resource constrained, and this is presenting additional challenges to the sometimes computationally expensive security solutions found in enterprise security systems. The development of energy-efficient security mechanisms is addressed in the contribution by Riecker et al. on "Lightweight Energy Consumption Based Intrusion Detection Systems for Wireless Sensor Networks". This work effectively utilises mobile agents to detect intrusions based on the energy consumption of sensor nodes.

In their contribution "Multi-operator Wireless Mesh Networks Secured by an All Encompassing Security Archi- tecture", Egners et al. address another important issue of wireless networks resulting from the interconnectedness of wireless systems. This is created by nodes frequently joining or leaving the network and being under the control of more than one operator. Focusing on mesh networks, this work advances the secure handover of mesh clients in a network of wirelessly connected mesh routers.

In "Verifying Multicast-Based Security Protocols", Martina and Paulson insist on today's facility of broadcasting a message by pressing a single button. Their work extends an established method of security protocol verification with the common operation of multicasting. As a result, more notational uniformity is possible because both unicast and broadcast can be formalised by means of the new multicast primitive. Also the expressiveness of the method is enhanced, thanks to the demonstrated feasibility of modelling protocols for secure electronic auctions.

In conclusion, our gratitude goes to all anonymous reviewers. Through a number of review rounds, we are confident that their tireless work appreciably improved the original submissions. We must also thank the authors for pursuing their work with determination and competence. All this makes us all the more proud of the contents we are delivering through this issue. 